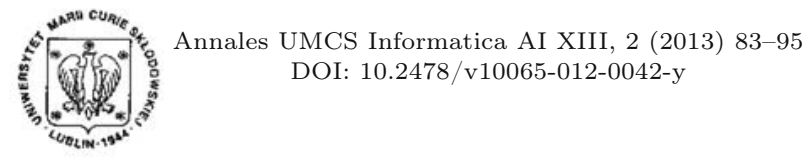

DOI: $10.2478 /$ v10065-012-0042-y
Annales UMCS

Informatica

Lublin-Polonia

Sectio AI

http://www.annales.umcs.lublin.pl/

\title{
Convergent traffic in the environment of wireless MESH technology
}

\author{
Andrzej Paszkiewicz ${ }^{1 *}$, Marek Bolanowski ${ }^{1 \dagger}$, Przemysław Zapała $^{1 \ddagger}$ \\ ${ }^{1}$ Rzeszów University of Technology, Faculty of Electrical and Computer Engineering, \\ Department of Distributed Systems, \\ al. Powstańców Warszawy 12, 35-959 Rzeszów, Poland
}

\begin{abstract}
The paper presents a performance study of wireless MESH networks in the context of the convergent transmission. Nowadays, the integration of voice, video and data is becoming the norm. However, the wireless LAN environment, and especially MESH technology is an unfriendly environment due to its characteristics. Therefore, the impact of the size of transmitted frames to delays in the network based on mesh technology was analyzed. The sizes of transmitted frames corresponded to standards for Voice over IP, Video over IP that are commonly used. Additionally, a model of effective load balancing in mesh networks was presented. Then, the reliability and flexibility of these networks will be increased.
\end{abstract}

\section{Introduction}

Nowadays wireless networks are becoming more popular. The construction of new structures and development of the already existing in many cases are based on wireless technology. This situation is a result, among others, of economic factors, because wireless infrastructure is usually cheaper and on the other hand, allows high mobility of ends users. Though transmission parameters and greater demands to ensure transmission security towards Ethernet networks, wireless network especially WLAN integrate into convergent services $[\mathbf{1}, \mathbf{2}]$. Therefore phone conversations, implementation of video conferencing or receiving stream video transmission in this type of network become standards. Convergent transmission requires to ensure adequate parameters

*andrzejp@prz.edu.pl

${ }^{\dagger}$ marekb@prz.edu.pl

${ }^{\ddagger}$ p.zapala@stud.prz.edu.pl 
especially in relation to response, Jitter, bandwidth etc. Therefore wireless networks are a difficult area for this type of services.

The progressive wireless network should be characterized by the mobility, flexibility, security, reliability. Furthermore, this network is expected by end users in job, home, during the holiday. Commonly used $802.11 \mathrm{a} / \mathrm{b} / \mathrm{g} / \mathrm{n}[\mathbf{3}]$ technologies with the use of proper mechanisms QoS meet these expectations in many cases. These solutions are based on BSS and EBSS topology [4]. However, it is possible to present many areas in which BSS and EBSS topologies are not sufficient. In these cases the integration of individual Access Points or some group is typically associated with their direct access to the Ethernet network. Despite possible security and effective end users roaming, the necessity of an accessibility by each access point to wired network is a major limitation and does not allow for effective use of the WLAN in many cases. An example of such application areas are parks, university campuses, monitoring network or dispersed sensor system and any other areas where it is impossible and unfavourably to use wired network. At present it is possible to refer to a developed concept of a broadband network for the Podkarpacie voivodship [5]. In this concept the scope of wired networks use and necessity of using wireless networks are clearly defined. These examples may constitute the basis for using of wireless networks of the Mesh type [6, 7]. These types of networks are defined by 802.11 s standard and allow to establish a wireless connection without necessity of applying the wired network to each access point. In many cases this is only one solution to ensure an access to network services in the selected areas.

In this paper the possibility of applying the WLAN networks, Mesh type for convergent transmission was analyzed. Additionally, in view of a lack of classical solutions mechanism load balancing in Mesh networks, a new solution based on available collection and acceptable path was presented. This approach allows for increasing effectiveness of the Mesh networks in respect to convergent transmission.

\section{MESH networks}

Mesh network is defined by IEEE $802.11 \mathrm{~s}$ standard $[\mathbf{6}, \mathbf{8}]$. This is a communication node network between access points. In this case there is no need to use the Ethernet network. All Mesh functionality is included in the data link layer which causes increase of Mesh efficiency because the node does not need to use a ISO/OSI's higher layer. Connection to the Ethernet network is realized on the edge of Mesh network. The following scheme shows demonstration of the Mesh network.

The structure of Mesh contains two types of access points: Mesh Portal and Mesh Point. Mesh Portal (MPP) is the gateway between the wireless Mesh network and the Ethernet network. However, Mesh Points functions are similar to the thin access points [7].

One of the fundamental assumptions of Mesh network is possibility of using a lot of potential paths to the transmission date. 802.11s standard uses Hybrid Wireless Mesh Protocol (HWMP) to route between nodes, which have two modes [9]: 


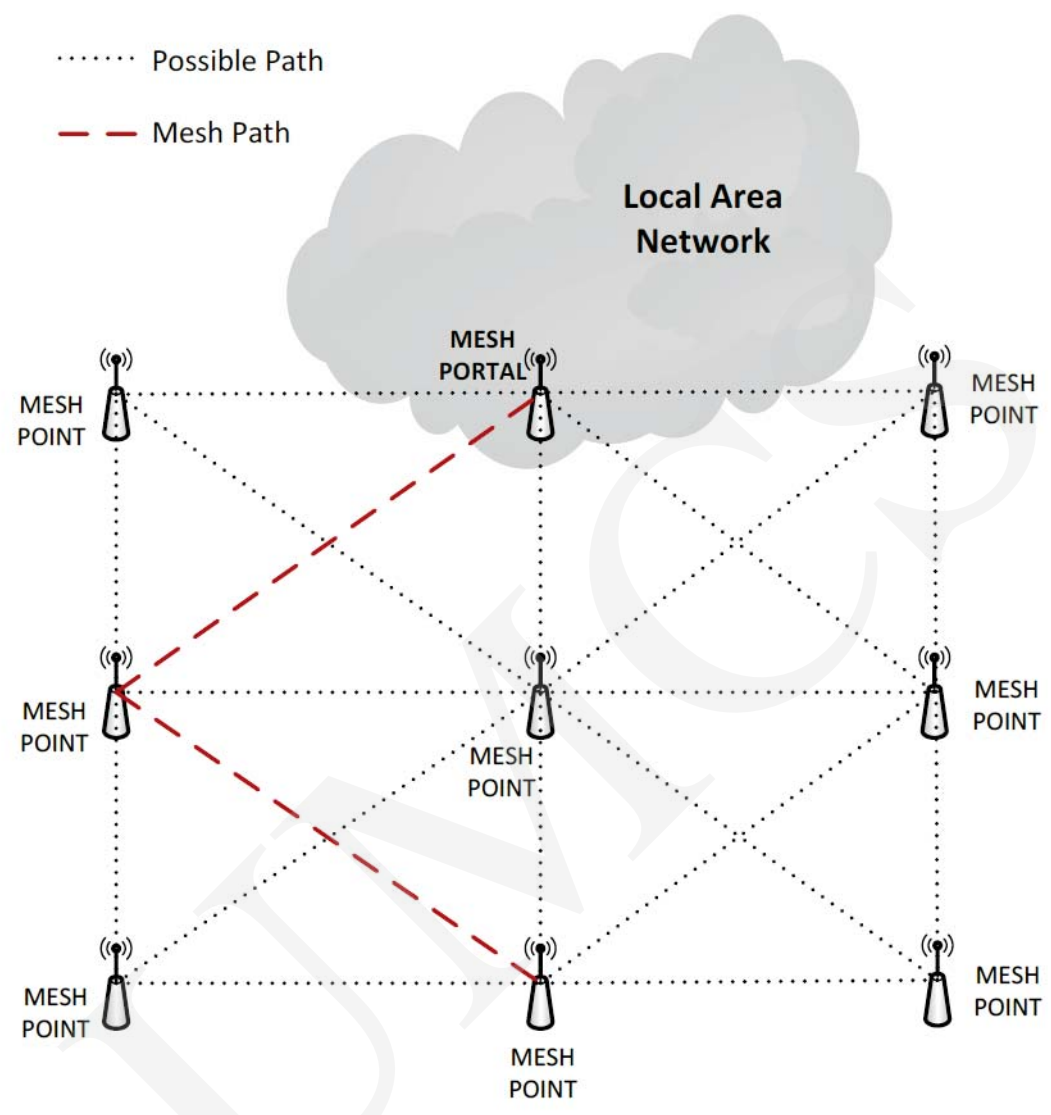

Fig. 1. Mesh network topology.

- Mode Proactive

- Mode on-demand (Reactive)

In the proactive mode all nodes transmit information about path as a link metric table. This creates a tree path in the Mesh network. This tree is composed of root and branch which cross a successive node up to the leaf. One of the nodes has to be configured as a root. The best solution is choice of the node performs as a gateway. When a tree path is created, all nodes in the network will know path to root and to another node in the network. While in the reactive mode, nodes use on-demand path selection. This method is always available in the network and works without configured Mesh portal. This allows all nodes in the network to communicate between each other by pear-to-pear path [3]. The protocol HWMP uses inter alia a sequence number to find old information about path. Packets are discarded if their sequence number is lower than currently used by node. It prevents the formation of routing loop [7]. 
Frame format which is used in the Mesh network is similar to the frame format used in 802.11 standard. Mesh frame has to forward more parameters and can contain six MAC addresses. The first four MAC addresses are contained in 802.11 standard header. The next two addresses are located in the Mesh control field. Nodes that are in the network, but they are not Mesh nodes can see the Mesh control field as part of the body frame.

Considering the usage of Mesh network to create infrastructure for convergent services, transmission possibility for this type of network should be carefully looked for. The feature of this type of network is the dependence real bandwidth on the Mesh path from the smallest element, so:

$$
C_{j}=\min \left(c_{j}^{i}\right)
$$

where $c_{j}^{i}$ is the bandwidth $i$-th hop on $j$-th path. During the analysis of the convergent network, communication between the last Mesh point and the end-of device should be considered as well. This link is not taken into consideration by 802.11 standard, because it just considers communication between the Mesh portal and the Mesh points. This situation is presented in Fig. 2.



Fig. 2. Throughput in mesh networks.

\section{Convergent networks and transmission parameters}

Convergent traffic includes data, voice and video. Typically, data are much less sensitive to a delay in contrast to transmission of voice or video. One of more important factors that are being considered during building packet voice networks is the proper capacity planning. Commonly used codecs for VoIP are characterized by the following values (Table 1) [10] for the Codec Sample Size (Bytes) and Codec Sample Interval $(\mathrm{ms})$ :

In order to determine the required bandwidth for each codec the following formula should be used [11]: 
Pobrane z czasopisma Annales AI- Informatica http://ai.annales.umcs.pl

Data: 26/04/2023 17:21:28

Table 1. Examples of Codec Sample Size and Codec Sample Interval for the VoIP codecs.

\begin{tabular}{|c|c|c|c|}
\hline Codec \& Bit Rate (Kbps) & $\begin{array}{c}\text { Codec Sample Size } \\
\text { (Bytes) }\end{array}$ & $\begin{array}{c}\text { Codec Sample Interval } \\
(\mathbf{m s})\end{array}$ & $\begin{array}{c}\text { Voice Payload Size } \\
\text { (Bytes) }\end{array}$ \\
\hline G.711 (64 Kbps) & 80 Bytes & $10 \mathrm{~ms}$ & 160 Bytes \\
\hline G.729 $(8 \mathrm{Kbps})$ & 10 Bytes & $10 \mathrm{~ms}$ & 20 Bytes \\
\hline G.723.1 (6.3 Kbps) & 24 Bytes & $30 \mathrm{~ms}$ & 24 Bytes \\
\hline G.723.1 (5.3 Kbps) & 20 Bytes & $30 \mathrm{~ms}$ Bytes \\
\hline G.726 $(32 \mathrm{Kbps})$ & 20 Bytes & $5 \mathrm{~ms}$ & 80 Bytes \\
\hline G.726 $(24 \mathrm{Kbps})$ & 15 Bytes & $5 \mathrm{~ms}$ & 60 Bytes \\
\hline G.728(16 Kbps) & 10 Bytes & $5 \mathrm{~ms}$ & 60 Bytes \\
\hline G722_64k(64 Kbps) & 80 Bytes & $10 \mathrm{~ms}$ & 160 Bytes \\
\hline
\end{tabular}

- Total packet size $=(\mathrm{L} 2$ header $)+(\mathrm{IP} / \mathrm{UDP} / \mathrm{RTP}$ header $)+($ voice payload size)

- $\mathrm{PPS}=($ codec bit rate $) /($ voice payload size $)$

- Bandwidth $=($ total packet size $) *$ PPS

On this basis, values of the transmission bandwidth are obtained (Table 2) [10].

Table 2. Bandwidth for VoIP codecs.

\begin{tabular}{|c|c|c|}
\hline Codec \& Bit Rate (Kbps) & $\begin{array}{c}\text { Bandwidth Ethernet } \\
\text { (Kbps) }\end{array}$ & $\begin{array}{c}\text { Bandwidth cRTP } \\
\text { (kbps) }\end{array}$ \\
\hline G.711 (64 Kbps) & $87.2 \mathrm{Kbps}$ & $67.6 \mathrm{Kbps}$ \\
\hline G.729 (8 Kbps) & $31.2 \mathrm{Kbps}$ & $11.6 \mathrm{Kbps}$ \\
\hline G.723.1 (6.3 Kbps) & $21.9 \mathrm{Kbps}$ & $8.8 \mathrm{Kbps}$ \\
\hline G.723.1 (5.3 Kbps) & $20.8 \mathrm{Kbps}$ & $7.7 \mathrm{Kbps}$ \\
\hline G.726 (32 Kbps) & $55.2 \mathrm{Kbps}$ & $35.6 \mathrm{Kbps}$ \\
\hline G.726 $(24 \mathrm{Kbps})$ & $47.2 \mathrm{Kbps}$ & $27.6 \mathrm{Kbps}$ \\
\hline G.728 (16 Kbps) & $31.5 \mathrm{Kbps}$ & $18.4 \mathrm{Kbps}$ \\
\hline G722_64k (64 Kbps) & $87.2 \mathrm{Kbps}$ & $67.6 \mathrm{Kbps}$ \\
\hline
\end{tabular}

Apart from Voice over IP the Video over IP is used as well. Video transfer in the network requires more bandwidth to ensure a minimum bit rate. This means that 
Pobrane z czasopisma Annales AI- Informatica http://ai.annales.umcs.pl

Data: 26/04/2023 17:21:28

convergent transmissions become more and more stringent for wireless networks. The example list of required transmission parameters for convergent traffic is presented in Table $3[\mathbf{1 2}]$.

Table 3. Summary of transmission parameters for a convergent traffic.

\begin{tabular}{|c|c|c|}
\hline Application & $\begin{array}{c}\text { Offered Load } \\
\text { (Mbps) }\end{array}$ & Max Delay (ms) \\
\hline SDTV & $4-5$ & 200 \\
\hline HDTV (Video/Audio) & $19.2-24$ & 200 \\
\hline DVD & 9.8 peak & 100 \\
\hline Video Conf & $0.128-2$ & 200 \\
\hline $\begin{array}{c}\text { Internet Streaming } \\
\text { video/audio }\end{array}$ & $0.1-4$ & 200 \\
\hline $\begin{array}{c}\text { Internet Streaming audio } \\
\text { VoIP }\end{array}$ & $0.064-0.256$ & 30 \\
\hline
\end{tabular}

Similarly to the voice transmission, specific video codecs characterized by appropriate parameters are used. This allows to reduce necessary bandwidth.

\section{Mesh network performance for convergent traffic}

In order to carry out analysis, the Mesh network structure was created. This network was tested for date, audio and video transmission parameters. In this case the parameters created earlier were used. The basic size determining maintenance of transmission is inter alia delay which should not exceed $30 \mathrm{~ms}$ for voice calls (e.g. VoIP). In the case of audio/video transmission of peek the maximum delay for e.g. video conferences should not exceed 100ms. Less demanding transmissions are e.g. streaming audio/video, which should not exceed 200ms. Considering inter alia the above assumptions, artificially generated transmissions and practical convergent transmissions were introduced to the network.

Fig. 3 and Table 4 confirm that the Mesh network can ensure proper infrastructure for convergent transmission. Minimal and average delay for transmissions was very low. Peek of delay values pertained to exceptional situations which during transmission are very rare and do not affect the transmission quality. Similarly, the ratio of the loss 
Pobrane z czasopisma Annales AI- Informatica http://ai.annales.umcs.pl

Data: 26/04/2023 17:21:28

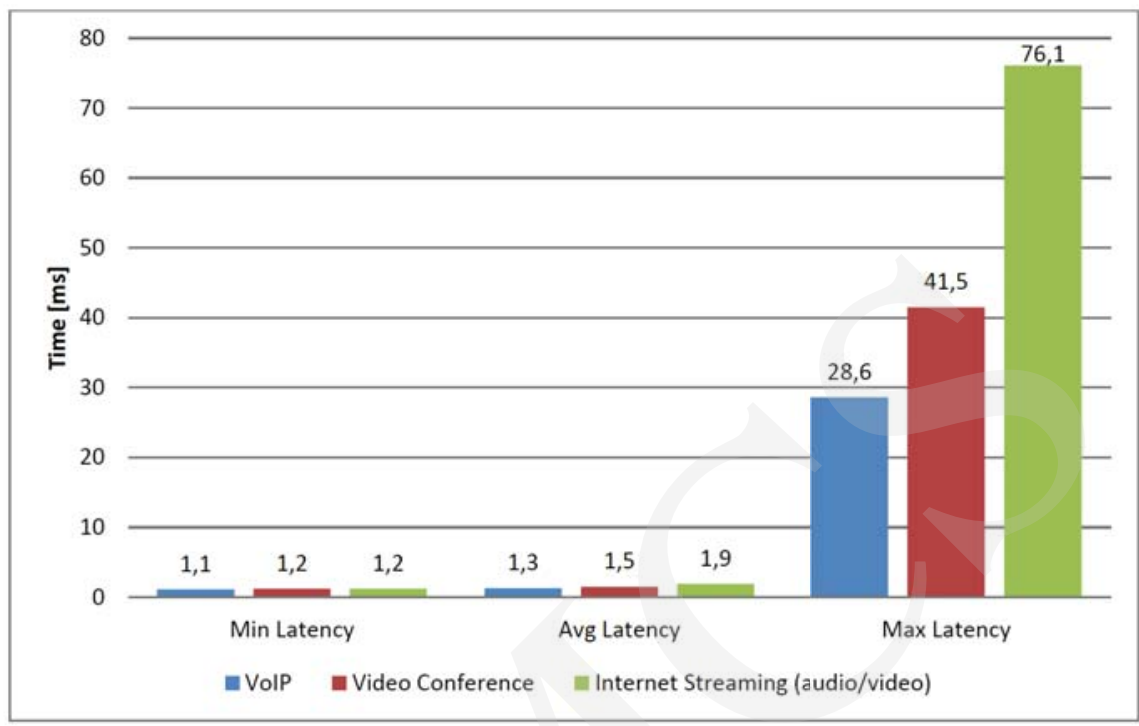

Fig. 3. Delays for a convergent traffic in the 3-nodal mesh network.

Table 4. Number of lost packets during transmission.

\begin{tabular}{|c|c|c|c|}
\hline & Transmited Packet & Lost Packet & Average Lost Rate [bps] \\
\hline VoIP & 24040320 & 0 & 0 \\
\hline Video Conference & 24144240 & 960 & 283 \\
\hline $\begin{array}{c}\text { Internet Streaming } \\
\text { (audio/video) }\end{array}$ & 24195840 & 1560 & \\
\hline
\end{tabular}

frames to the transmitted frames is very low almost negligible. After analysing the frame size and packet per second necessary for the VoIP transmission, conducting simulation of many voice calls was possible. On the assumption that one voice call requires 50PPS and frame of size 200Bytes it is possible to verify how the network will behave when 60 simultaneous voice calls exist. The results of these tests are presented in Fig. 4.

Average delay proportionally increasing to a number of calls results from their support by each device. However, the maximum Jitter confirms the situations which may in a very violent way lead to the degradation of transmission parameters and thereby maintaining the connections is impossible. This situation can be a result of interferences, collisions, or path selections in the Mesh network. 
Pobrane z czasopisma Annales AI- Informatica http://ai.annales.umcs.pl

Data: 26/04/2023 17:21:28

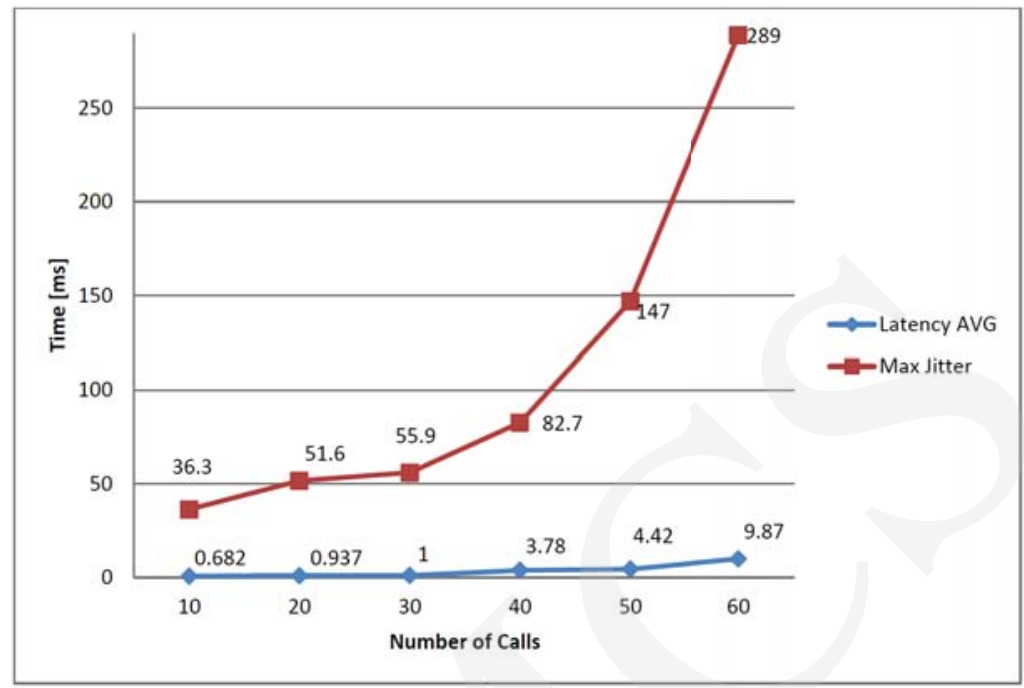

Fig. 4. Delay and jitter depending on the number of connections.

The studies also show the relation between the number of hops and delay. This dependence is described as the number of nodes transmitted by packet travels. This is a distance between a receiving node and a transmitter node. This simulation is a multiple run (Fig. 5). The connection between nodes was established on 54Mbps in 802.11a standard. The average of the relationship delay time of packet to the number of hops was examined. Frames for the size 56Bytes (Fig. 6) and 1500Bytes (Fig. 7) were transmitted. The size of frames is the result of the analysis available protocols and codecs for convergent transmission. Tested topology and transmission parameters ensure high quality transmissions for voice and video.

As can be appreciated along with each hop increasing response time for packets (Fig. 5). However, this time does not threaten to break connection sessions. The obtained results confirm possibility of using Mesh network for convergent transmission. In Fig. 5 actual transmission on each connection between the nodes can be also seen. This figure shows the actual path bandwidth depending on the bandwidth of the slowest links from the Mesh network.

\section{The proposed new solution}

Analyzing the obtained results for both unreal generated traffic and real Voice over IP and Video of IP traffic should be noted that MESH technology can be used to provide convergent services. However, there is a lack of additional mechanisms to support this type of traffic in a hostile WLAN environment. Quality and the number of connections can dramatically decrease in the case of high interference. Of course, in that case there is a possibility to use for example, a classic QoS for wireless LAN based 
Pobrane z czasopisma Annales AI- Informatica http://ai.annales.umcs.pl

Data: 26/04/2023 17:21:28



Fig. 5. Average Bandwidth and Response Time.



Fig. 6. Delay for packet size 56 Bytes.

on 802.11e [4].This standard introduces only four access classes of network traffic. This is much less than in the wired networks. There are other solutions that guarantee the necessary bandwidth for traffic such as Voice Call Admissions Control [13]. However, this solution can be used at the end of the path in the mesh network to support roaming in order to guarantee the necessary bandwidth for phone calls.

Taking the above information into account, there should be proposed a solution that would increase effectively the use of available network MESH. For this purpose, there is proposed a solution for using load balancing for each available path in the mesh structure. LACP [14] and ECMP [15] known for wired solutions cannot be applied 
Pobrane z czasopisma Annales AI- Informatica http://ai.annales.umcs.pl

Data: 26/04/2023 17:21:28



Fig. 7. Delay for packet size 1500 Bytes.

here. LACP cannot be used in itself because it relates to the node-to-node connections. Whereas, we deal with the path in the mesh where multiple nodes can be. Another solution is ECMP that provides load balancing on equal route to the same destination. However, an equal fixed routes is hard to ensure in the mesh network.

The proposed solution is based on the alternative, acceptable mesh paths. Fig. 8 shows a diagram of the use of these paths.

The proposed solution consists of several modules, each of which is responsible for other actions.

- Paths determinationbetween the portal and the specific (end) mesh point. Available mechanisms in the MESH technology (such proactive or relative mode) can be used for this purpose. Then it is possible to obtain a set of available paths $P_{i j}=\left\{p_{i j}^{k}\right\}$ between the $i$-th mesh portal and the $j$-th mesh point.

- Selection of acceptable mesh pathsin the set $P_{i j}$. For this purpose, it is necessary to define appropriate parameters. Of course, the available number of hops can be used, but we suggest defining a set of approval parameters $V_{i j}^{k}=\left\{C_{i j}^{k}, D_{i j}^{k}, J_{i j}^{k}, E_{i j}^{k}, H_{i j}^{k}\right\}$, where $C_{i j}^{k}$ represents a real capacity of the $k$-th mesh path, $D_{i j}^{k}$ is the average delay on the path, $J_{i j}^{k}$ denotes the maximum delay variation (jitter), $E_{i j}^{k}$ is the error rate expressed as a percentage of a number of lost packets and $H_{i j}^{k}$ refers to the maximum acceptable number of hops. Due to the need to determine the current value of each parameter an interval, e.g. 5 seconds will be specified. The result of this part of the mechanism is a subset $P_{i j}^{\prime} \in P_{i j}$, whose elements meet the constraints resulting from the approval parameters.

- Hashing Function. This function is responsible for load balancing by directing conversations to each individual, acceptable mesh path (Fig. 9). Flow (conversation) is 




Fig. 8. The concept of acceptable MESH paths.

defined by the source and destination address as well as the identifier of the applicationlayer protocol such as RTP, NEO, SMTP, etc. This approach allows to specify a given conversation.

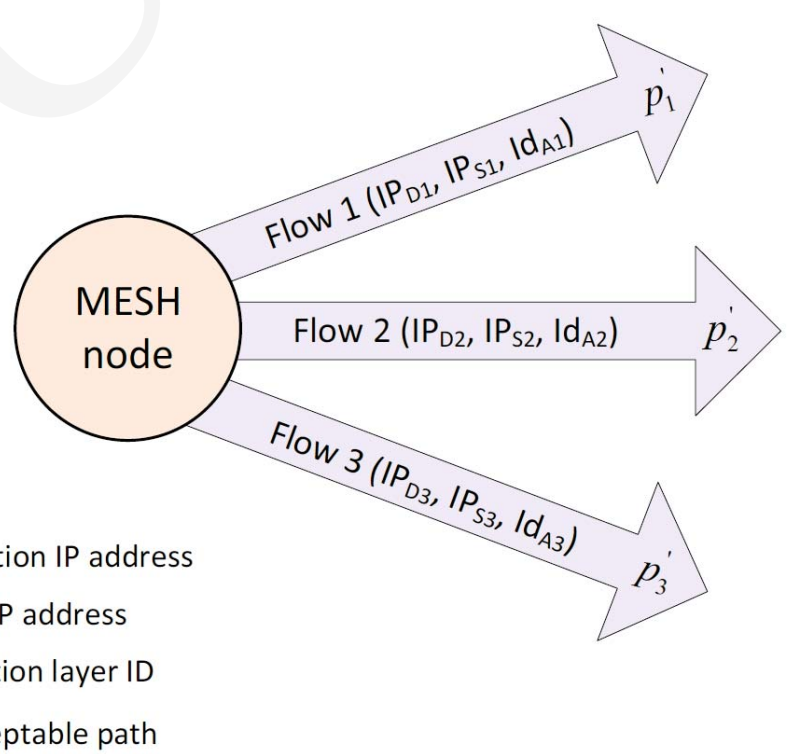

Fig. 9. Hashing Function - Load Balancing. 
- Permanent analysis of available and acceptable paths module. This module is responsible for permanent analysis of available and acceptable mesh paths. At the time of detection of new paths, they are added to the set $P_{i j}$. Similarly, in the case of paths that no longer meet the requirements of $V_{i j}^{k}$, they will be removed from the set of acceptable paths. Due to the fact that convergent traffic has special high demands to ensure stable transmission parameters, it may turn out that the current mesh path will no longer exist or its performance will drop to an unacceptable value. Then there must be the possibility of forwarding one conversation to the other alternative path. There is no problem when resources are available but when resources are limited, it is difficult to keep resources availability. This is achieved by the takeover module.

- The takeover moduleis responsible for keeping a specific part of the bandwidth on each of the acceptable mesh paths (Fig. 10). This value should be configurable and allow to takeover the conversation traffic for which the mesh path was removed or does not meet required parameters.

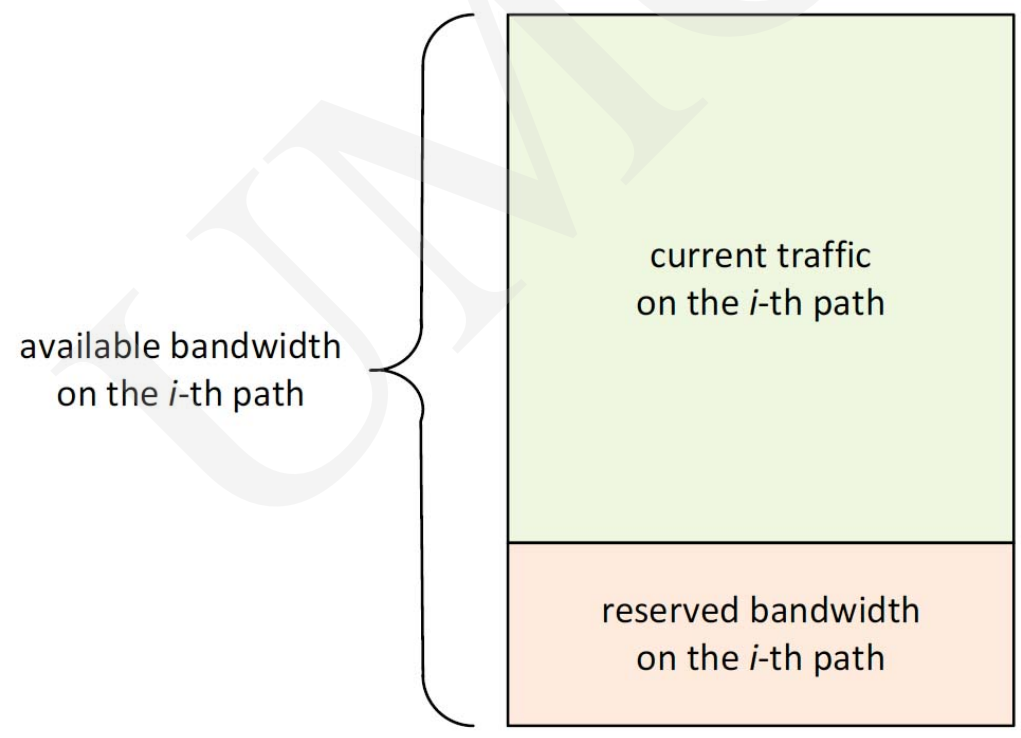

Fig. 10. Bandwidth reservation on the path.

The proposed solution does not interfere with the QoS mechanism available in 802.11e in any way. On the contrary, it can fully coexist with it. That means that individual conversations can also obtain appropriate access categories.

\section{Conclusions}

The possibility of using mesh network for handling convergent traffic was analyzed in the paper. This traffic is sensitive particularly to the available bandwidth, delay, etc. 
Mesh network concept is not an obstacle to this type of implementations. However, it does not ensure a specific support for this type of traffic as well.

Tests clearly show that in the case of the lack of additional mechanisms in mesh technology e.g. the change of the current path causes significant delays and that can lead to a break of a communication session or significantly worsen the quality of transmission in many cases.

The proposed solution seems to be a reasonable. It also uses available mechanisms and introduces new mechanisms in the mesh and allows mesh to be more efficient structures.

\section{References}

[1] Donoso Y., Network Design for IP Convergence, Auerbach (2009).

[2] Hanrahan H., Network Convergence: Services, Applications, Transport, and Operations Support, John Wiley \& Sons, England (2007).

[3] Sendra S., Fernandez P., Turro C., Lloret J., IEEE 802.11a/b/g/n Indoor Coverage and Performance Comparison, Wireless and Mobile Communications (ICWMC), 2010 6th International Conference, Valencia (2010).

[4] Roshan P., Leary J., 802.11 Wireless Lan Fundamentals, Cisco Press (2006).

[5] Opracowanie zbiorowe pod redakcją M. Stroińskiego, Studium rozwoju sieci szerokopasmowej dla województwa podkarpackiego na lata 2007-2013, Ośrodek Wydawnictw Naukowych, Poznań (2008).

[6] Hiertz G. R., Denteneer D., Max S., Taori R., Cardona J., Berlemann L., Walke B., IEEE 802.11s: The WLAN Mesh Standard, IEEE Wireless Communications 17 (1) (2010).

[7] Camp J. D., Knightly E. W., The IEEE 802.11s Extended Service Set Mesh Networking Standard, IEEE Communications Magazine 46 (8) (2008).

[8] Lee M. J., Zheng J., Young-Bae Ko, Shrestha D. M., Emerging Standards For Wireless MESH Technology, IEEE Wireless Communications 13 (2) (2006).

[9] Sgora A., Vergados D. D., Chatzimisios P., IEEE 802.11s Wireless Mesh Networks: Challenges and Perspectives, Social Informatics and Telecommunications Engineering 13, Springer Berlin Heidelberg (2009).

[10] Voice Over IP - Per Call Bandwidth Consumption, Cisco Website.

[11] Voice Support on Aruba Controllers and Remote Access Points for Fixed Telecommuter Deployments, Aruba Networks Website.

[12] Rahrer T., Fiandra R., Wright S., Triple-play Services Quality of Experience (QoE) Requirements, Technical Report TR-126, DSL Forum (December 2006).

[13] Perros H. G., Elsayed K. M., Call admission control schemes: a review, IEEE Communications Magazine 34 (11) (1996)

[14] IEEE Std 802.1AX-2008 IEEE Standard for Local and Metropolitan Area Networks — Link Aggregation, IEEE Standards Association (2008).

[15] Hopps C., Analysis of an Equal-Cost Multi-Path Algorithm, Network Working Group (2000). 